\title{
Research on Simulation of Neuro-fuzzy Control Strategy of Eddy Current Retarder ${ }^{(1)}$
}

\author{
Yu Mingjin ${ }^{\mathrm{a},{ }^{* 1}}$, Qiu Xuyun ${ }^{\mathrm{a},{ }^{* 2}}$, Zhang Liangxiu ${ }^{\mathrm{a},{ }^{* 3}}$, Huang Yong ${ }^{\mathrm{b}}$ \\ ${ }^{a}$ Automotive Department, Shandong Jiaotong University, Jinan, China \\ ${ }^{b}$ College of Mechanical Engineering, Chongaing University, Chongqing, China
}

\begin{abstract}
A simulation model of eddy current retarder is designed, including $1 / 4$ vehicle model, eddy current retarder model, tire model. Based on the PID and neuro-fuzzy controltechnique, twokinds of control algorithm on constant speed control heavytruckare designed. On the built simulation system, the control algorithm is simulated and test. The results show that the neuro-fuzzy controller can implement high precision and strong robust control effects on control of eddy current retarder system.
\end{abstract}

Index Terms: Eddy Current Retarder; Heavy Truck Model; Neuro-fuzzy Control

(C) 2011 Published by MECS Publisher. Selection and/or peer review under responsibility of the International Conference on E-Business System and Education Technology

\section{Introduction}

Eddy Current Retarder(ECR) is a device that transfers kinetic energy generating by vehicle into thermal energy which will emit afterwards. Based on electromagnetic theory, ECR can realize deceleration and retardation. It can keep the main brake remaining in the cooling state in order to work effectively in emergencies. The use of retarder can significantly reduce the wear of the main brake, extend life expectancy to 4-10 times the original, which will greatly reduce operating costs, improve the usability of vehicle.

Most of the currently-existing retarder adopts hierarchical control relays[1], so the braking torque generated is hierarchical and not continuous. Thus the braking torque generated differs from the actual demands, hard to meet the braking requirements[2].

In this paper the neuro-fuzzy control technique is utilized to develop a new stepless retarder control method. A retarder and vehicle model is built to simulate the process. The result shows that the neuro-fuzzy control method can achieve better eddy current retarder stepless control and make vehicles go downhill smoothly at a constant speed, and have strong robustness and adaptability.

\footnotetext{
${ }^{(1)}$ Fund aid: Western Transport Construction and Technology plan projects(2009318000072), Shandong province traffic Department project (2008Y024 (6)), Shandong Jiaotong University Research Fund project (z201006).

Corresponding author:

| E-mail address: ${ }^{* 1}$ shdymj@163.com; ${ }^{* 2}$ qiuxuyun@163.com; ${ }^{* 3}$ zhangliangxiu@163.com
} 


\section{Simulation Model of ECR}

\subsection{Vehicle Model}

According to the requirement of simulation, a $1 / 4$ vehicle kinematic model in ramp way is built, and rolling resistance is neglected during braking. Assuming the vehicle goes straight, without considering the problem of tire lateral force, the longitudinal dynamics equation is:

$$
F_{j}=F_{i}-F_{f}-F_{w}
$$

Where $F_{j}$ is vehicle deceleration resistance, $F_{i}$ is sliding force, $F_{f}$ is adhesion, $F_{w}$ is air resistance.

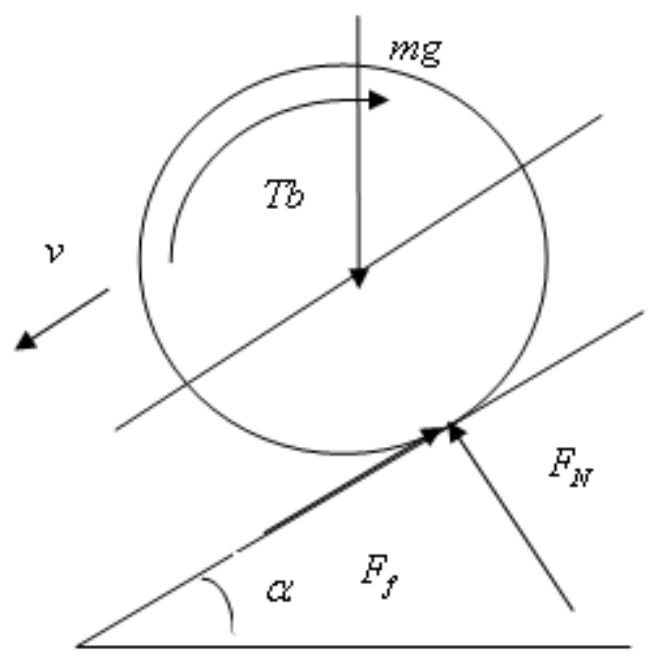

Fig. 1. Single wheel truck model in rampway

Vehicle deceleration resistance, sliding power, traction, air resistance can be calculated by the following formula:

$$
\begin{aligned}
& F_{j}=-M \dot{v} \\
& F_{f}=\mu M g \\
& F_{w}=\frac{C_{d} A v_{a}^{2}}{21.15} \\
& F_{i}=M g \sin \alpha
\end{aligned}
$$


Where ${ }^{\mu}$ is the adhesion coefficient between the wheels and the ground, which is a function of tire slip ratio, $\mu=f(s) . C_{d}$ is air resistance coefficient. $v_{\text {is vehicle speed. }} \omega$ is angular velocity for the wheels. $\alpha$ is ramp angle. $M$ is the vehicle quality.

Tire model under stress of heavy truck in braking:

$$
J \dot{\omega}=T_{b}-F_{f} r
$$

Where $J$ is the rotational inertia of the wheels, $\omega$ is the angular deceleration of the wheels, $F_{f}$ is rolling resistance, $T_{b}$ is the braking torque effected on the wheels, $r$ is the radius of the wheels.

$$
T_{b}=T_{\omega} \cdot i_{0}
$$

$T_{\omega}$ is the retarder braking torque, ${ }^{i_{0}}$ is the drive ratio of the final reducer in transmission.

\subsection{The dynamic model of ECR}

Braking torque is the main standard measured of eddy current retarder. However, it's a very difficult task to accuratly calculate the braking torque. Its institutional structure is not very complicated, but the braking torque is affected by many factors. Currently, the calculate of eddy current retarder braking torque adopt a method of combining simplifying and testing calculation. In this paper, on the basis of access to reference[3], the formula of calculating of eddy current retarder braking torque can be expressed as follows:

$$
\begin{aligned}
& T_{1}=90 \sqrt{2} N_{p}\left(\rho \mu_{0}\right)^{\frac{2}{3}}(N I)^{2} \pi d^{4} \sqrt{\omega} \\
& T_{2}=\sqrt{\mu_{r}} \arcsin \left(\frac{d}{2 R_{l}}\right)\left(16 \pi \rho l_{\varepsilon}+\sqrt{2} k_{e} \mu_{0} \sqrt{\frac{2 \rho}{\omega \mu_{0} \mu_{r}}} \frac{\pi d^{2}}{4} \omega\right)^{2} \\
& T=\frac{T_{1}}{T_{2}}
\end{aligned}
$$

Where $k_{e}$ is conversion coefficient, which usually $k_{e}=1.5 \rho$ is resistivity of disk, $\rho=1.5 \times 10^{-7} \Omega \cdot m . \sigma$ is conductivity of disk. $\mu_{0}$ is the vacuum permeability, $\mu_{0}=4 \pi \times 10^{-7} \mathrm{H} / \mathrm{m} . N$ is the excitation coil turns, taken 320. $\mu_{r}$ is relative permeability of disk, low carbon steel is usually taken 200. $\omega$ is angular frequency for the magnetic field. ${ }^{N_{p}}$ is the pole pairs. $l_{\varepsilon}$ is air gap, taken $1.4 \mathrm{~mm}$. 


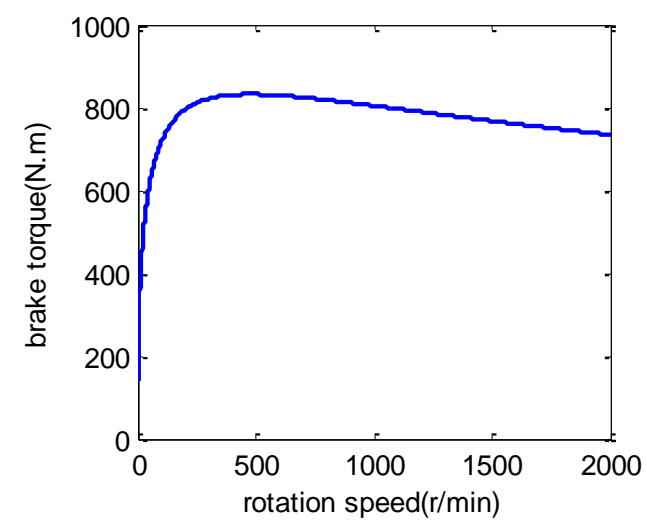

Fig. 2. Brake torque of ECR model

$$
d \text { is core diameter, } d=101.5 \mathrm{~mm} .
$$

\subsection{Tire Model}

Tire model adopts the magic formula to calculate the tire lateral force and aligning torque, the magic formula is shown as follows:

$$
Y(x)=D \sin (C \arctan (B x-E(B x-\arctan (B x))))
$$

Where $\mathrm{Y}$ can be the longitudinal force, which can also be a lateral force or aligning torque, etc. the variables $\mathrm{X}$ can express the longitudinal slip rate or slip angle of the tire,respectively, under different circumstances.B, C, $\mathrm{D}, \mathrm{E}$ are determined successively by the tire vertical load and camber angle, which are the constant related with the surface. By changing these parameters, different road adhesion coefficient can be simulated. The parameters in this magic formula used in this paper are shown as follows:

$\mathrm{D}=0.9, \mathrm{C}=2.2, \mathrm{~B}=6.0, \mathrm{E}=0.98$.

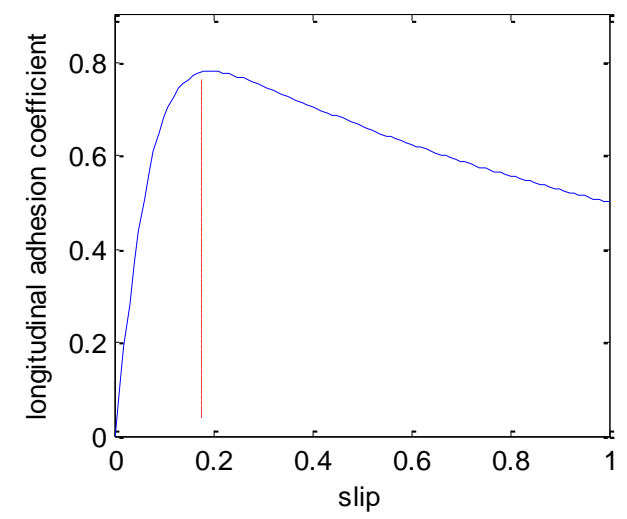

Fig. 3. Longitudinal adhesion coefficient 


\section{Retarder Control Strategy}

Adaptive Neuro-Fuzzy Inference System in the MATLAB is a system which combines the neural network theory with T-S fuzzy reasoning[4] .It can be built the fuzzy Inference system (FIS) with an adaptive modeling method ,based on plently of date, because of the establishment of the FIS using neural network is the result of data processing.

At first ,the PID control is the method to control data in this article, and then take the results as the training data, according to the algorithm provided by MATLAB to calculate and built a two-dimensional T-S model. The vehicle speed deviation and speed deviation change rate is selected as the input and the excitation current as the output to realize the control of the constant speed when vehicles go downhill .The neural fuzzy control structure shown in Fig.4 .

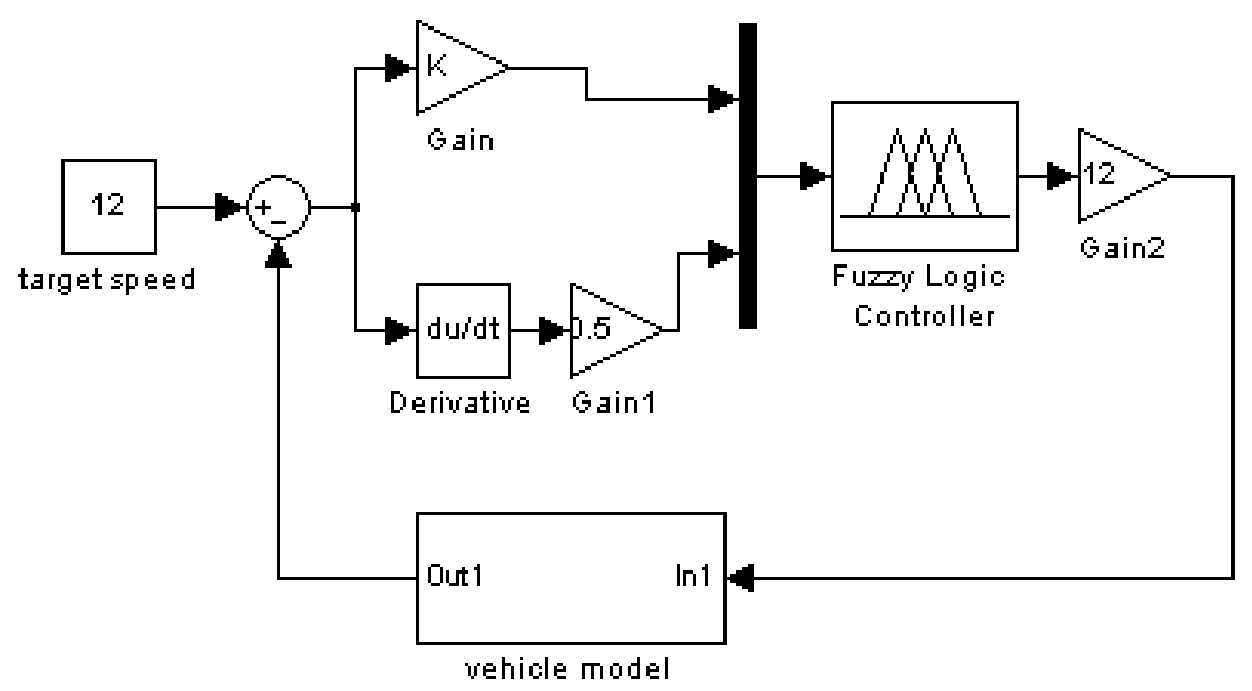

Fig. 4. Neuro-fuzzy controller

Through neural network training, there are three fuzzy rules:

if $\mathrm{e}$ is $\mathrm{e} 1$ and $\mathrm{ec}$ is ec 1 then

$$
u 1=-11.65 u 1-5.821 u 2+15.62
$$

if $\mathrm{e}$ is $\mathrm{e} 2$ and $\mathrm{ec}$ is ec 2 then

$$
u 2=-11.41 u 1-5.908 u 2+15.94
$$

if $\mathrm{e}$ is $\mathrm{e} 3$ and $\mathrm{ec}$ is ec 3 then

$$
u 3=-11.42 u 1-5.879 u 2+15.44
$$

\section{Ramp Constant Speed Control Simulation Model}

According to the requirement of simulation[5], a vehicle ramp constant speed control model which shown in the Fig.5 is built, mainly including PID and neuro-fuzzy control model, eddy current retarder model, tire model, 1 / 4 vehicle model.

Eddy current retarder is expressed as(8-10), tire model adopt the magic formula (11), PID and fuzzy neural controller is the two-step controlled or also be individually controlled. 


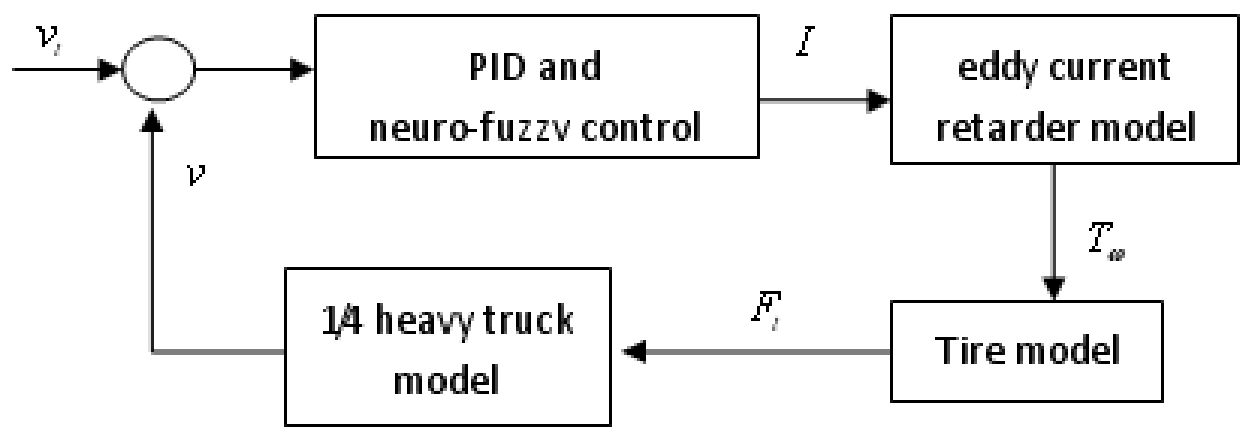

Fig. 5. Simulation model of heavy truck with ECR

\section{Simulation Results and Analysis}

In the MATLAB / simulink simulation circumstances, the constant control of the vehicle simulation in rampway model is built. Set up vehicles and the eddy current retarder simulation parameters are as follows: $m=7000 \mathrm{~kg}, r=0.52 \mathrm{~m}, J=20 \mathrm{~kg} \cdot \mathrm{m}^{2}, A=6 \mathrm{~m}^{2}, i_{0}=5.833, \eta=0.85, C_{d}=0.3$, the results shown as follows:

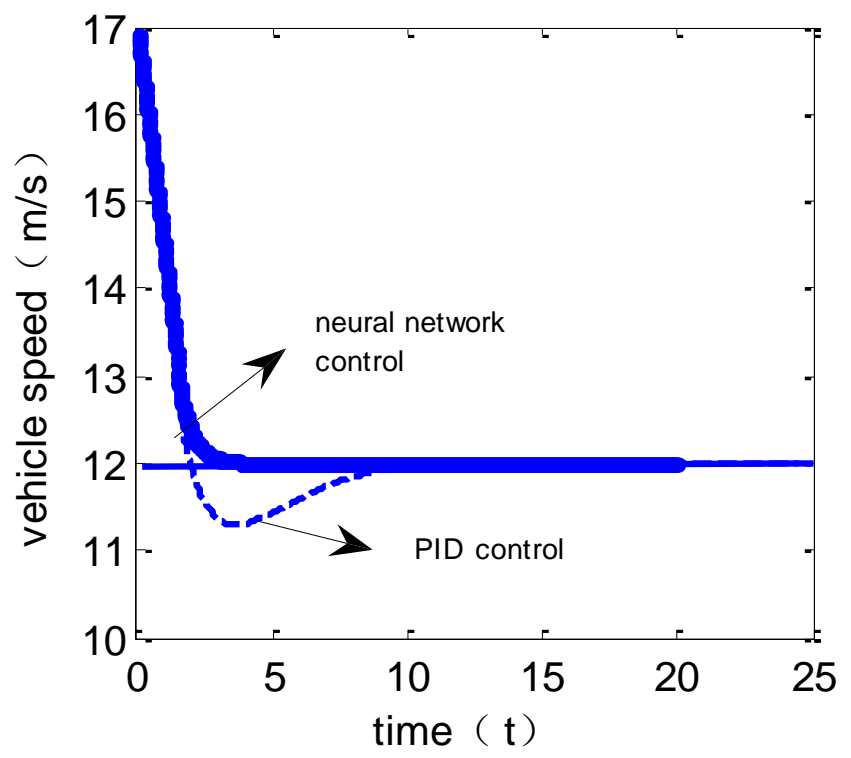

Fig. 6. The comparison between neural network control and PID control 


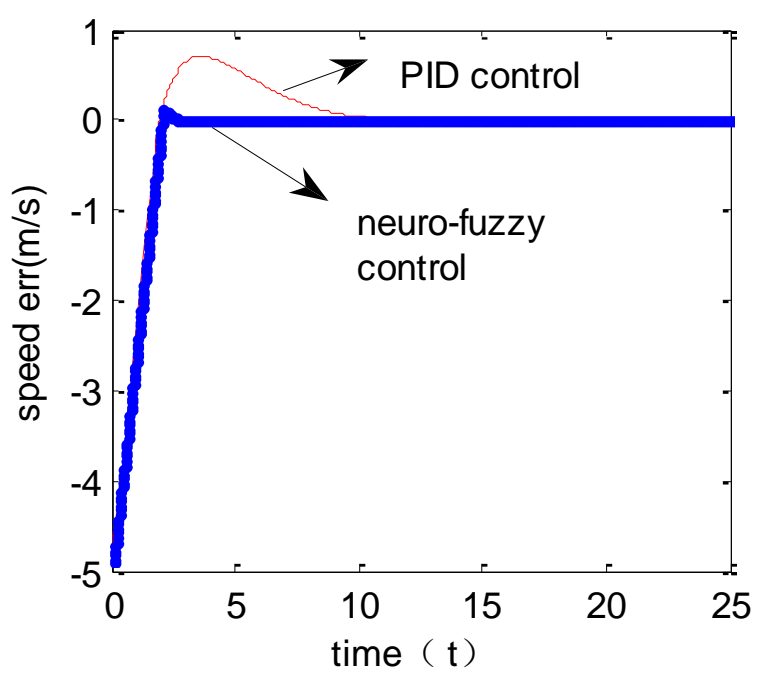

Fig. 7. The change of deviation in the two control method

A conclusion can be drawn that it is appropriate to achieve constant speed control of the eddy current retarder in braking when go downhill. This smart constant speed control can improve vehicle's safety and driver's comfort.

\section{Acknowledgements}

The thesis has got the support from Western Transport Construction and Technology plan projects (2009318000072) in the Transport ministry, Shandong province traffic Department project (2008Y024 (6)) ,Shandong Jiaotong University Research Fund project (z201006)'s funding. Thanks to the Ministry of Transport and Shandong province traffic Department for the support to the paper.Meanwhile in the writing process, the thesis cites a large number of references, here I want to express my appreciation to the writer of reference.

\section{References}

[1] Lai Nanhui. Wu Liming. Wang Guitang.Research on Automotive Eddy Current Retarder and Its Virtual Testing and Simulation ICEMI ,2007.3:166-169. (in Chinese)

[2] Sun Weimin. Eddy Current Retarder Theory. Master Thesis of Beijing University of Technology, 2005. (in Chinese)

[3] He Ren, Yi Feng Yan, He Jianqing. Calculate of Eddy Current Retarder Braking Torque , Automotive Engineering. 2004 (2) :197-200. (in Chinese)

[4] Shi Xin Min, HaoZhengqing. Fuzzy Control and MATLAB Simulation. Tsinghua University Press, 2008. (in Chinese)

[5] Ding Nenggeng, Yu Guizhen. Vehicle Dynamics and Control. Harbin Engineering University Press. 2009. (in Chinese) 